\title{
STRESSER-PÉAN Guy y Claude, Tamtok, sitio arqueológico huasteco. 2 - Su vida cotidiana
}

Dominique Michelet

\section{OpenEdition \\ Journals}

Édition électronique

URL : https://journals.openedition.org/jsa/10615

DOI : 10.4000/jsa. 10615

ISSN : 1957-7842

Éditeur

Société des américanistes

Édition imprimée

Date de publication : 20 décembre 2008

Pagination : 229-232

ISSN : 0037-9174

\section{Référence électronique}

Dominique Michelet, « StResser-PÉAn Guy y Claude, Tamtok, sitio arqueológico huasteco. 2 - Su vida cotidiana », Journal de la Société des américanistes [En ligne], 94-2 | 2008, mis en ligne le 20 avril 2009, consulté le 04 septembre 2022. URL : http://journals.openedition.org/jsa/10615 ; DOI : https:// doi.org/10.4000/jsa. 10615

Ce document a été généré automatiquement le 4 septembre 2022

Tous droits réservés 


\title{
STRESSER-PÉAN Guy y Claude, Tamtok, sitio arqueológico huasteco. 2 - Su vida cotidiana
}

\author{
Dominique Michelet
}

\section{RÉFÉRENCE}

STRESSER-PÉAN Guy y Claude, con dibujos de Françoise Bagot, Tamtok, sitio arqueológico huasteco. 2 - Su vida cotidiana, CONACULTA/INAH, Instituto de Cultura de San Luis, Fomento cultural Banamex A. C., CEMCA, México, 2005, 480 p., bibl., tabl., 75 figures au trait, 30 pl. photo N\&B, 5 coul., 3 doc. hors texte + cédérom avec version française

1 Ce second volume, consacré aux travaux archéologiques dirigés par Guy Stresser-Péan sur le site de Tamtok dans les tout premiers temps -1962-1964- de la Mission archéologique et ethnologique française au Mexique (aujourd'hui Centre français d'études mexicaines et centraméricaines, CEMCA), est dans le droit fil du premier tome paru en 2001 et dont le Journal de la Société des Américanistes avait rendu compte (voir vol. 87, pp. 409-412, 2001). Même luxe éditorial sur tous les aspects déjà signalés, renforcé d'ailleurs ici par l'utilisation d'un beau papier glacé - mais on ne saurait se plaindre, par exemple, de la multiplication des dessins, bien plus nombreux en réalité que les figures dûment numérotées, et régulièrement répétés, semble-t-il chaque fois que les auteurs jugeaient que cela était utile pour la lecture; même parti pris de présentation de l'information sous une forme fortement segmentée, mais clairement hiérarchisée - les quatre parties de ce tome se décomposent en vingt-deux chapitres qui, le cas échéant, peuvent avoir moins de cinq pages ; même souci d'exhaustivité dans la présentation des données...

2 Après que le premier volume eut traité du site en général, que Stresser-Péan fut le premier à étudier et à fouiller, ainsi que des fouilles importantes qu'il y réalisa, ce nouvel ouvrage est dédié à l'ensemble du matériel recueilli, fondamentalement par la fouille 
d'une tranchée de $124 \mathrm{~m}$ de long et $4 \mathrm{~m}$ de large sur la pente ouest de la plus large éminence du site (connue comme la «Loma de Paso del Bayo » dans les années 1960 et appelée aujourd'hui « Cubilete»), celle de 26 sondages stratigraphiques répartis en divers points de l'établissement, et le dégagement des 23 édifices qui composent le Groupe A, dégagement qui s'est accompagné souvent de sondages à l'intérieur des plates-formes. De façon on ne peut plus classique, l'étude aborde successivement l'industrie céramique (récipients, figurines et objets divers), le matériel lithique (en silex, en obsidienne, en basalte, à quoi est ajoutée la statuaire), puis le métal, les coquillages et les restes osseux, animaux et humains.

3 La volumineuse section qui concerne les récipients en céramique pourra certainement dérouter plus d'un spécialiste d'aujourd'hui. Dans la totalité du matériel qui avait été récolté, Claude et Guy Stresser-Péan ont fort bien identifié qu'il existe des composantes représentatives de deux complexes distincts, séparés dans le temps par plus ou moins huit siècles : un lot, minoritaire, de pièces et de fragments remontant probablement à la fin du Classique ancien, aux alentours de 500-600 apr. J.-C., et un ensemble, beaucoup plus conséquent et complet, de productions qu'ils datent eux-mêmes $d u x^{e}$ siècle. Mais qu'on ne cherche pas dans ces pages une définition claire de ces complexes, ni même des informations chiffrées sur les provenances, notamment stratigraphiques, des types et sous-types qui sont décrits et extraordinairement illustrés. Ici, la «typologie » est presque un instrument de pure présentation des quelque 51000 tessons et 222 pièces qui constituent le corpus. Un cas extrême pourrait être la distinction de deux sous-types dans la catégorie Zaquil rouge, avec un premier rattaché à la première phase d'occupation et le second au Postclassique; mais on cherchera en vain dans le texte les critères qui distinguent les deux. À la critique plus ou moins implicite contenue dans ce que l'on vient d'écrire, on doit toutefois apporter deux nuances. L'analyse qui a été faite du matériel a été précédée d'un énorme travail de remontage, ce qui a permis aux auteurs, et nous permet à nous aujourd'hui, d'identifier, sur profils complets, les formes exactes d'un très grand nombre de récipients, la variété de celles-ci et, le cas échéant, la diversité et la complexité des décors. Autre point fort du travail publié, et nous l'avons déjà mentionné, le caractère systématique de l'illustration et son excellence (merci François Bagot!) offrent à tous ceux qui sont ou seront à l'avenir intéressés par la production céramique de la région aux périodes représentées une source d'information incomparable dont les différents éléments pourraient même peut-être être ré-analysés et restructurés. Toujours concernant les récipients en céramique, on ne peut pas manquer enfin de souligner que l'attention portée à la reconstitution des formes débouche, bien souvent, sur des commentaires très bienvenus sur la fonction des différents vases. D'une façon générale, ici comme dans le reste de l'ouvrage, ce que les auteurs proposent, c'est moins un traitement canonique des objets qu'une paléo-ethnographie sur ces derniers. Dans cette perspective, on est d'ailleurs un peu déçu que l'abondance des spécimens de vases miniatures (143 des 222 pièces " complètes ») ne débouche pas sur une discussion plus approfondie. De même, pour les petits vases dits « en forme de creusets » qui ne sont pas rares dans la catégorie Pâte grossière, on note, sur les dessins fournis, les formes, plutôt répétitives, et l'épaisseur relativement élevée des parois et on apprend par le texte qu'ils portent des stigmates d'exposition au feu, mais aucune hypothèse fonctionnelle n'est avancée à leur sujet.

4 Le matériel lithique, qui occupe la deuxième section du livre et la quatrième partie de l'ouvrage complet, est traité d'une façon tout autant exhaustive que la céramique. Ainsi, les 114 pointes de projectiles découvertes (dont 6 en obsidienne) sont toutes illustrées 
dans le volume, ce qui pourra sans doute autoriser, comme on le suggérait plus haut, des analyses nouvelles sur cette collection. Pour ce qui est des pointes et couteaux bifaces en silex, une des données les plus notables tient aux contextes de leur apparition : ils ont été trouvés, en effet, en majorité dans des sépultures, notamment celle, qui était multiple, de la structure AC2 (71 pointes), ou dans des offrandes votives. L'obsidienne, de son côté, qui est censée provenir du gisement de Zacualtipan (Hidalgo), pour la noire, et du Cerro de Las Navajas, pour la verte, paraît, à l'exception de quelques pièces, avoir été nettement plus liée à des usages domestiques. En outre, deux aires de taille d'obsidienne noire ont été localisées, tandis que, sur le site comme dans beaucoup d'autres lieux, tout porte à croire que l'obsidienne du Cerro de Las Navajas est parvenue sous forme de produits finis. Quelques lignes sur les statuts qui pourraient avoir été associés à l'une et l'autre des catégories d'obsidienne sont, comme plusieurs apparentes courtes digressions qui parsèment l'ouvrage, tout à fait stimulantes: la différence de couleur n'aurait pas eu forcément un sens symbolique fort (contrairement aux oppositions sur lesquelles les populations paraissent avoir bel et bien joué entre plusieurs silex et l'obsidienne), mais une signification d'abord sociale: la possession d'objets en obsidienne verte, coûteuse, aurait été l'apanage, et donc une des marques distinctives, des familles les plus importantes.

Dès l'époque des fouilles de Stresser-Péan, Tamtok s'est révélé être un site remarquable par sa statuaire. Un constat qu'a, bien sûr, considérablement renforcé la découverte de nouvelles sculptures par le projet que dirigèrent Patricio Dávila et Diana Zaragoza en 1994-1995 - les deux pièces principales trouvées par ces derniers sont, avec leur autorisation, illustrées et commentées dans l'ouvrage des Stresser, mais on peut aussi consulter Patricio Dávila C. et Diana Zaragoza O. (« Tantoc : una ciudad en la Huasteca », Arqueología mexicana, 54, pp. 66-69) et celle, en 2005, par Guillermo Ahuja O., d'une formidable dalle à reliefs mesurant près de $8 \mathrm{~m}$ de long et $4 \mathrm{~m}$ de haut (voir à ce sujet Arqueología mexicana, 79, p. 14, 2006). Les trois pièces de découverte plus ou moins récente que l'on vient d'évoquer sont datées par leurs inventeurs du Préclassique, un jugement que rien, au moins dans ce qui a été publié, ne permet de soutenir. En réalité, il paraît bien plus logique - et confirmé d'ailleurs par le dépôt d'érection qui accompagnait les stèles 1 et 2, mis au jour par Stresser-Péan -, de considérer, à l'instar de ce dernier, que là aussi sont représentées les deux périodes que la céramique signalait : le Classique, plutôt moyen, et le Postclassique récent. Remarquons seulement au passage que l'importance de la statuaire classique indique l'existence, à Tamtok et à cette époque, d'un établissement important doté d'un rôle politico-religieux fort.

Objet en métal (un seul), coquillages, restes osseux animaux et humains sont étudiés et présentés par Guy et Claude Stresser-Péan avec le même souci du tout-dire et... du détail. Et c'est avec la partie finale et ses deux chapitres, dont le premier s'intitule de façon quasi emblématique « La vie et la religion à Tamtok dans le siècle qui précéda l'arrivée des Espagnols", que l'on comprend le véritable enjeu des centaines de pages qui précèdent : faire revivre, à partir de tous les éléments matériels récupérés, inventoriés, décrits et donnés à voir, le site dans un maximum de ses aspects. Les choix opérés au moment du terrain ont fait que ce que Guy et Claude Stresser-Péan reconstituent le mieux, c'est le centre d'un village du dernier siècle préhispanique, occupé par une élite, assez modeste, mais une élite quand même. Comme le reconnaissent les auteurs, la population ordinaire leur a largement échappé. Cependant il faut aussi ajouter qu'aujourd'hui beaucoup d'éléments nouveaux montrent que le Tamtok classique a, lui, 
sans aucun doute été un centre huastèque majeur, ce que les fouilles des années 1960 permettaient tout juste d'entrevoir.

\section{AUTEURS}

\section{DOMINIQUE MICHELET}

UMR 8096, Maison de l'archéologie et de l'ethnologie, Nanterre 